\title{
Artificial Companion: building a impacting relation
}

\author{
Sylvie PESTY \\ UMR 5217 - Laboratory LIG, University of Grenoble, France \\ sylvie.pesty@imag.fr \\ Dominique DUHAUT \\ Université de Bretagne Sud, France \\ dominique.duhaut@univ-ubs.fr
}

\begin{abstract}
In this paper we show that we are in front of an evolution from traditional human-computer interactions to a kind of intense exchange between the human user and new generation of virtual or real systems -Embodied Conversational Agents (ECAs) or affective robotsbringing the interaction to another level, the "relation level". We call these systems "companions" that is to say systems with which the user wants to build a kind of lifelong relationship.

We thus argue that we need to go beyond the concepts acceptability and believability of system to get closer to human and look for "impact" concept. We will see that this problematic is shared between the community of researchers in Embodied Conversational Agents (ECAs) and in affective robotics fields. We put forward a definition of an "impacting relation" that will enable believable interactive ECAs or robots to become believable impacting companions.
\end{abstract}

\section{Introduction}

At the present time, human-computer interactions are turning to intense exchanges between the human and the system, which is no longer a simple interface of graphical objects, but a much more complex one: an "embodied system". This embodied system is either virtual Embodied Conversational Agents (ECAs)- or real affective robots. In these embodied systems, expressive, affective, interactive and multimodal aspects (gestures, facial expressions, gaze...) are essential. It brings the traditional human-computer interaction to another level: the "relation level". Generally this new generation of systems is called: "companions" that is to say systems with which the user wants to build a kind of life-long relationship.

In this paper, we will briefly overview the traditional human-computer interactions. We will explain why we need to go beyond the concepts of system acceptability and believability to get closer to the concept of "impact". We will first propose a definition of a notion called "bringing together" and then define what a "relationship" between two "identities" is. We thus propose to overcome the concept of interaction between a human and a companion robot or a companion ECA to go towards the concept of "impacting relation". Finally, the last section of this paper presents some preliminary experiments to illustrate our claims.

\section{In the beginning ...}

In human-computer interactions the first level of interaction is the interface one where the basic interactions are direct manipulations of graphical objects using the mouse in a window [1]. The typical applications used in this context are, for instance, text editing, calculation, data management, computer aided design, etc.

At this level, the evaluation of the quality of the interaction is principally based on the usefulness of the system. One of the most cited models is the one of Davis et al. [1], the Technology Acceptance Model (TAM) for predicting and studying user acceptance of any technology. In 1993, Nielsen [2] proposed a model of system acceptability (figure 1); he distinguished the practical acceptability and the social acceptability. He described the practical acceptability that focused on the relationship between functionalities of the technology and ease of use. It thus embraces the usefulness and usability. 


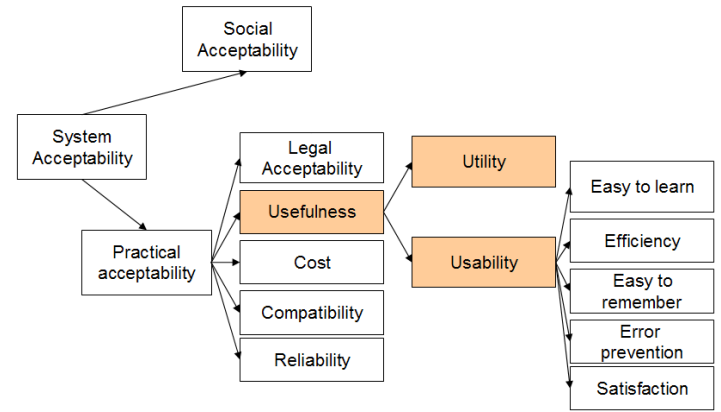

Figure 1: J. Nielsen model of system acceptability [2]

In a second level of human-computer interactions, the usefulness of the interface is no longer the central notion, but the interaction becomes the fundamental point. This interaction is studied in four dimensions.

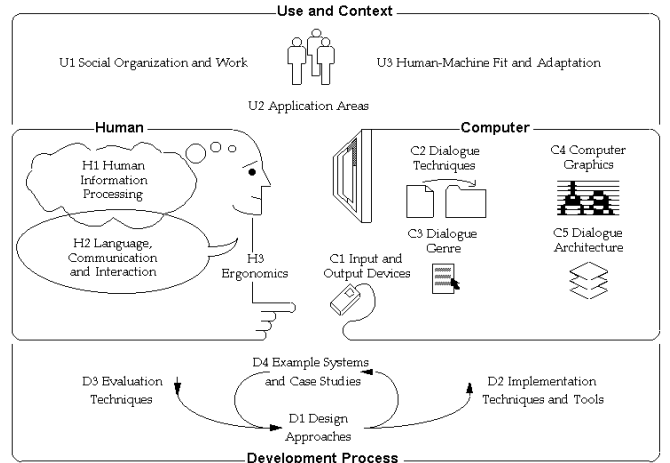

Figure 2: Human-Computer Interaction [3]

HCI is characterized by:

- Use and context of computers: human social organization and work, application areas, humanmachine fit and adaptation

- Human characteristics: human information processing, language, communication, interaction, ergonomics

- Computer system and interface architecture: input and output devices, dialogue techniques, dialogue genre, computer graphics, dialogue architecture

- Development process: design approaches, implementation techniques, evaluation techniques, example systems and case studies

As stated by [4] from a robotic perspective, working with robots needs "to make automation components into effective "team players" when they interact with people in significant ways". Ten challenges are proposed to involve four basic requirements:

- Enter into an agreement, called a basic contract,

- That the participants intend to work together

- Be mutually predictable in their actions

- Be mutually directable

- Maintain common ground
The ten challenges proposed by [4] are:

1. Forming and maintaining the basic contract

2. Forming and maintaining adequate models of others' intentions and actions

3. Maintaining predictability without hobbling adaptivity

4. Maintaining adequate directability

5. Effective signalling of pertinent aspects of status and intentions

6. Observing and interpreting signals of status and intentions

7. Engagement in goal negotiation

8. Autonomy and planning technologies that are incremental and collaborative

9. Attention management

10. Controlling the costs of coordinated activity

Here again, we can notice that the system is shown under performance and efficiency criteria, which are very deterministic. The mutual predictability protects from surprises coming either from the activity of the system or from the human.

From the point of view of acceptability of the robot by the human, these challenges are by a majority linked to social acceptability as developed by Ajzek [5] (figure 3).

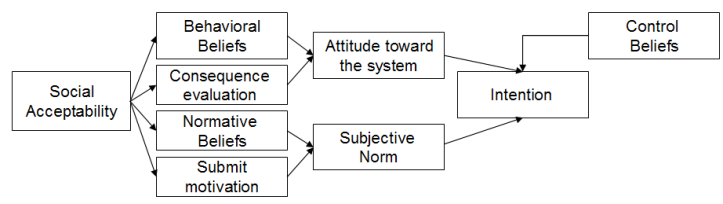

Figure 3: I. Ajzek theory of planned behaviour [5]

Social acceptability is no doubt significant, but if we go ahead, and turn to "companions", that is to say robots or Embodied Conversational Agents (ECAs) with which the user wants to build a kind of life-long relationship, it is not sufficient and we must go beyond this concept of system acceptance.

Companion robots and companion agents both represent a new form of interaction with machines. Ideally, human users simply use natural language, and these companions, either virtual or physical, are endowed with refined communication capabilities and emotional expressivity. They are more natural, and closer to the human user. For ECA researchers, believability is a central concept. ECAs must be believable or credible; the most general of these terms is used to describe anything we accept as true, even in the absence of absolute proof. As stated by Bates [9], believability includes the appearance of reactivity, goals, situated social competence and emotions. In this regard, believable ECAs need both rational and emotional skills in order to exhibit a coherent and natural behaviour. 
Ortony, 2003, said that a major issue is to make an emotional agent a believable emotional agent. The same for companion robots that need to exhibit a coherent and natural behaviour and need to be "affective robots".

Therefore, the challenge for robot researchers and ECA researchers is the same: to build affective interacting companions which are not just practically and socially acceptable, but believable. But what about the interaction between the companion and the user? Even if the companion has refined communication capabilities and multimodal emotional expressivity, is it sufficient? Wedo not think it is, and we propose to overcome interaction and communication to go towards the concepts of impacting relation.

In [6], C.Breazhal said that if the robot becomes a social object then it must include several capabilities. We generalize their propositions to our companions:

- Companions must have social-cognitive skills. Understanding people in social-psychological terms means appreciating the goals, beliefs, feelings, motives, and other mental states underlying human behaviour.

- Companions must communicate with humans to build relationships with people that provide mutual benefits over an extended period of time. The communication must be done in a simple language for humans.

- Companions must learn through imitation and tutelage, to learn new skills, knowledge, and tasks while on the interaction.

- The context of heavy (significant) interaction [7] between the human and the companion introduces constraints on psychological aspects, such as stress or pleasure.

- The predictability constraints on the behaviour of the companion must be relaxed to allow the human to have fun with the companion. By having fun we understand that the companion is able to act in a surprising way and therefore generates a corresponding emotion in the human.

As will be shown, they are no longer sufficient when considering significant human-system interaction. By significant interaction we mean interaction between a human and an intelligent real or virtual system which changes the quality of human life: for instance at home for disabled people. In this case, the problem is not to perform a task but to "feel good". This interaction introduces a new dimension which is no longer functional, but psychological. The issue then is creating an intelligent system that is "socially credible" (figure 4).

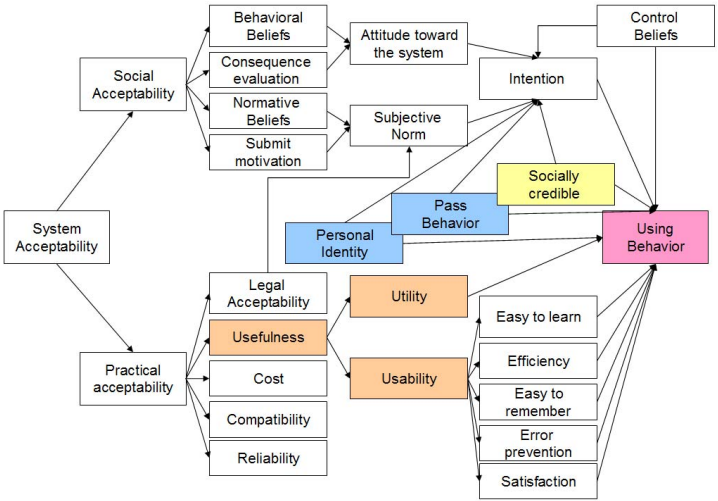

Figure 4: full model of human acceptability for systems with significant interaction [7].

In [7], we introduced the concept of "significant interaction" between a human user and a real or virtual system and the concept of "social credibility" (figure 4). It comes with the notion of believability that was well described in $[8,9]$. It is then necessary to deal with the personal identity and the history of the person that has already been described in works on social psychology, but also to build an intelligent system, which can be perceived as a social creature having its own identity and personality.

\section{From interaction to relation}

In this section, we want to clarify few definitions. We define the notion of "relation" based on "the bringing together" notion. This last notion will allow us to give a place to the general notions of emotion and personality usually used distinctly.

\subsection{Bringing together}

When defining the "bringing together" notion, it is important to describe the following four steps: how, where, who, what.

First, it is necessary to describe how the presence of a human and a robot is initiated. The notion of "state" is defined by the three different ways that two entities can initiate their relationship:

- Representation is when one can get information on the other one without being "physically" in front of him. This can appear if one looks to something produced by the other one: a text, a WEB, a drawing...

- Observation is when one can see the other one, but he does not know that he is being observed.

- Interaction is when one is getting information from the other by direct contact either by physical presence or by mediated support. 


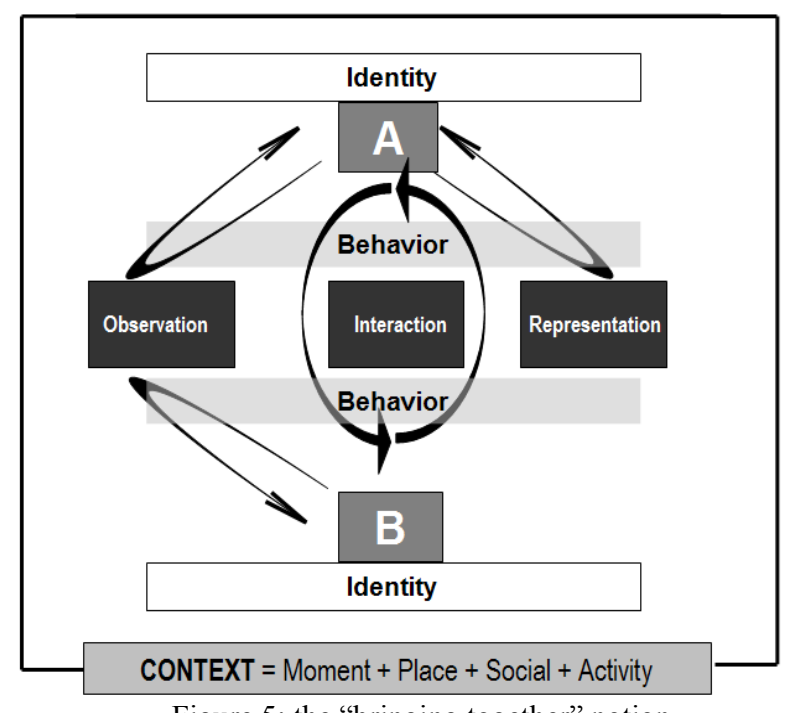

Figure 5: the "bringing together" notion

The second step is to describe where the action of bringing together is done. For this, we define the notion of context. The context is described with four elements:

- Moment: time of the presence, is it for a long /short time, was it expected or unexpected ...

- Place: the location impacts the behaviour of the person: a restaurant or a church change the rules of interaction between people.

- Social: is the one-one presence in presence of a group which impacts the distance between people, the way to speak and to act with respect to codes of this group

- Activity: the one-one presence is included in a task to be performed together. In the lower case, the task is only a meeting with no specific goal or outcome. Usually people meet others with a goal that the one-one presence tries to reach.

The third step is to describe the who, that is to say the identity of each participant. The notion of identity is based on three items:

- Knowledge: characterizes the things that a person shares with the other,

- Person: describes the attributes proper to one person and that does not change in the time (or change slowly)

- Lifestyle: describes the way that the person acts over the time.

Figure 6 gives details about these three different items.

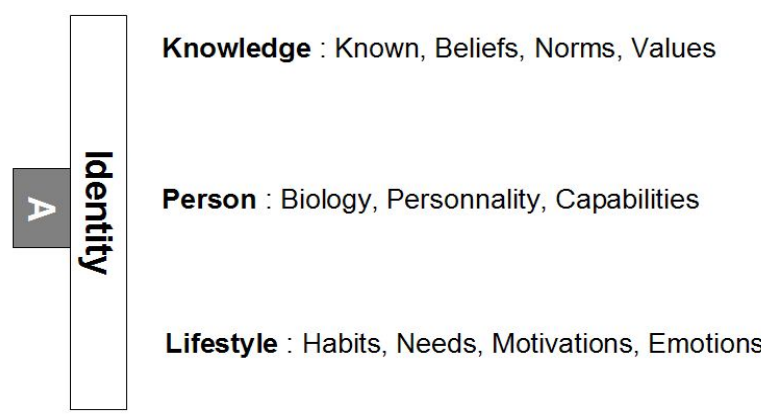

Figure 6: Definition of an identity

The last part is to describe the what part of the "bringing together" notion. The behaviour is the set of actions performed by an identity in a context. These actions are observed by the other identity depending on the state: observation, interaction and representation. It has two main objectives, a task to solve or an exchange with a human:

- Act to reach a goal or an outcome in the environment defined by the context

- Communication to exchange with the other one. Communication is described by the verbal and non-verbal aspects.

With these four steps, it is possible to propose a definition to the "bringing together" notion for two participants (two identities see figure 5):

Bringing together is the state in which two identities are in presence in a specific context where they have a specific behaviour.

\subsection{Building a relationship}

We now want to focus on a one-to-one interaction occurring between only two identities. When the interaction occurs over long periods of time, then a relationship appears and becomes visible or noticeable [10].

A relationship can be analysed (qualified) either by an external observer looking to the relationship of two identities $A \& B$ either by one of the two participants, A or B. We thus defined a relationship:

$A$ relationship between $A \& B$ is the modifications in $A \& B$ resulting from a repeated action of bringing together $A \& B$.

Let us now look atthe different elements that build a relationship. Hinde [12] proposed to study relationships according to ten dimensions: (1) content, (2) diversity, (3) quality, (4) pattern, (5) reciprocity and complementarity, (6) conflict and power, (7) self-disclosure, (8) 
interpersonal perception, (9) commitment, and (10) satisfaction.

From a social point of view, the anthropologists [10] have suggested four models of relationships: communal sharing (all group members are treated equally and share a common identity), authority ranking (people attend to each other's status in a hierarchical order), equality matching (the principle of quid pro quo predominates) and market pricing (people rationally weigh the utility of their behaviour in achieving outcomes in interaction). In [10], the authors also explained that one of the specific consequences of relationship is interdependence. The term interdependence refers to the manner in which two individuals influence each other's outcomes in the course of their interaction. For a companion robot or a companion ECA a long interaction is more than a day. The interaction includes the entire amount of time while they are in front of the individual. What is expected is that the relation constructed between the companion and the human brings to the human a positive feeling. This means that the companion must be able to know how its behaviour in the world will be perceived by the human, and what impact its behaviour will have on the emotional characteristics of the human.

Based on [11] we know that it is actually relevant to consider one unique measure qualifying emotions, which is "impact" instead of the traditional arousal, and valence measures.

We therefore would like to introduce the notion of "impacting relation" based on the positive feeling and impact.

$A$ relationship between $A \& B$ is an impacting relation for $A$ if the relationship with $B$ brings positive affective experiences to $A$ in a given context.

\section{First experiments on impact}

In this section, we report some preliminary experiments on a robot and an ECA to illustrate our claims.

We first use Greta [13], an ECA able to express its emotion through several modalities (verbal and nonverbal modalities). We built a protocol based on the evaluation of two expressive dialogue acts, apologise and rejoice, through a scenario played by the agent.

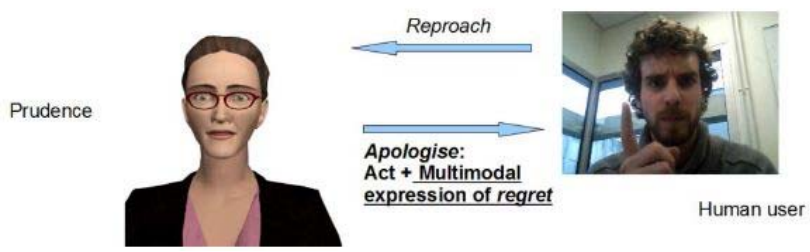

Figure 7: Measuring impact through sincerity and credibility with Greta [14]

The first hypothesis we wanted to verify was as follows: "the addition of non-verbal aspects consistent with the verbal aspects expressed by an ECA increases its sincerity and credibility". The results of the 23 users are in line with this hypothesis. The second part of the protocol consisted in a questionnaire whose aim was to collect the user's impressions, regarding the impact of the agent.

The results [14] show that the impact of the nonconsistent expression when Greta apologies or rejoices was higher than the impact of the consistent expression. Most users described Greta as "funny" or "hypocritical" or "making fun of them". One can thus imagine that an ECA endowed with humour or irony would have more impact on the user and would be able to build a life-long relationship with its user.

The second experiment is built with the same protocol [14], but instead of using Greta we use the robot "Bioliod".
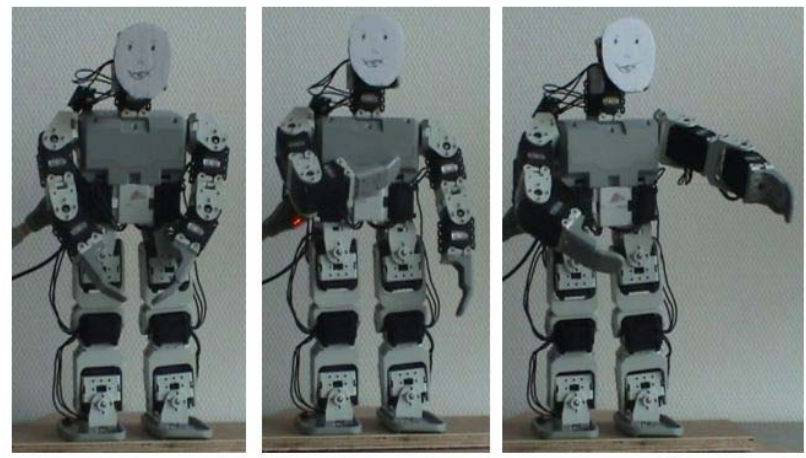

Figure 8: Measuring impact with the "bioloid" robot

The experiment was done with 60 children (10-15 years), 17 girls and 43 boys. We can conclude that the impact of the robot, built with sincerity and credibility criteria, is better if the gestures of the robot associated to the sentences are well-grounded.

A full description of this experiment is presented in [15]

\section{Conclusion}

In this paper we have presented why the concept of system acceptability and the concept of interaction must 
be overcome when we consider interactions between a human and an embodied system like a robot or an Embodied Conversational Agent (ECA). We put forward a definition of an "impacting relation" that will enable believable interactive ECAs or robots to become believable impacting companions able to build a kind of life-long relationship with the human. We have presented some preliminary experiments that have shown how a companion agent or a companion robot could have more or less of an "impact" on its user.

\section{References}

[1] Davis, F.D., 1989. Perceived usefulness, perceived ease of use, and user acceptance of information technology. MIS Quarterly 13, 319-339

[2] Nielsen, J., 1993. Usability Engineering. Academic Press, New-York.

[3] Brad A. Myers, A Brief History of Human Computer Interaction Technology Carnegie Mellon University School of Computer Science Technical Report CMU-CS96-163, dec 1996

[4] Gary Klein \& all, Ten Challenges for Making Automation a "Team Player" in Joint Human-Agent Activity, IEEE Intelligent Systems 2004

[5] Ajzen, I., 1991. The theory of planned behavior. Organizational Behavior and Human Decision Processes 50, 179-211.

[6] S. Coradeschi, \& al (2006) Human-Inspired Robots. IEEE Intelligent Systems 21(4), 74-85

[7] Pesty, S and Duhaut, D (2011) Acceptability in interaction - From robots to Embodied Conversational Agent, Int conf on computer graphics theory and applications GRAPP 2011, 5-7 march Algarve Portugal.

[8] Ortony A (2003), On making believable emotionaml adents believable, In R. Trappl, P. Petta \& S.Payr (Eds), Emotions in humans and artefacts, Cambrodge, MA: MIT Press

[9] J.Bates (1994) The role of emotion in believable agents, Communications of the ACM 37, 122-125

[10] Berscheid, E and Reis, H (1998) Attraction and close relationships, The handbook od social psychology ISBN0-19-521376-9 193-281.

[11] Ewbank M.P, Barnard P.J \& all (2009) The amygdale response to images with impact, SCAN 4, 127133.

[12] Hind R. (1996) Describing relationships, The diversity of human relationships, Cambridge university press

[13] Poggi, I., al. (2005) Greta: A believable embodied conversational agent.

[14] Riviere J., Adam C., Pesty S., Pelachaud C., Guiraud N, Lorini E, Longin D. (2011) Expressive Multimodal Conversational Acts for SAIBA agents, Proccedings of the 11th international conference on Intelligent Virtual Agents.

[15] C.Jost \& all (2011), Etude de l'impact du couplage geste et parole sur un robot, - Intercompréhension - de l'Intraspécifique à l'Interspécifique, Bretagne, $25 \& 26$ novembre (submitted) 